\title{
Carcinoma bem diferenciado de tireoide: perfil epidemiológico, resultados cirúrgicos e resposta oncológica.
}

\section{Well-differentiated tireoid carcinoma: epidemiological profile, surgical results and oncological response.}

Ricardo Mai Rocha, ACbC-eS1; Maria Carmen lopes Ferreira Siva Santos²; Carlos Musso²; Marco Homero de Sa Santos'; Marcelo lemos de-Almeida'; Gustavo Peixoto Soares Miguel, tCBC-ES'

\section{R E S U M O}

\begin{abstract}
Objetivo: conhecer o perfil epidemiológico dos pacientes submetidos à cirurgia do carcinoma bem diferenciado da tireoide no Hospital Universitário Cassiano Antônio Moraes da Universidade Federal do Espírito Santo, assim como os resultados oncológicos e as principais complicações pós-operatórias. Métodos: estudo transversal e retrospectivo de pacientes portadores de carcinoma bem diferenciado da tireoide operados no período de janeiro de 2008 a dezembro de 2015. Resultados: no período do estudo, dos 353 pacientes submetidos a tratamento cirúrgico da glândula tireoide, 95 eram portadores de CBDT. O carcinoma papilífero da tireoide foi o mais frequente $(91,57 \%)$. A tireoidectomia total não associada a esvaziamento cervical foi o procedimento cirúrgico mais frequente $(65,26 \%)$. As complicações pósoperatórias ocorreram em $6,31 \%$ dos pacientes, sendo o hematoma a mais frequente. O tempo médio de seguimento foi de 36,9 meses. A recidiva ocorreu em quatro pacientes $(4,21 \%)$, sendo locorregional em todos os casos. Os fatores prognósticos analisados, como sexo, idade, tamanho do tumor, acometimento linfonodal, estadiamento, tipo de cirurgia, histologia e iodoterapia complementar não demonstraram significância estatística. Conclusão: o carcinoma papilífero da tireoide foi a neoplasia maligna mais frequente, acometendo o sexo feminino na faixa etária dos 49 anos mais frequentemente. A recidiva locorregional ocorreu em quatro pacientes. O hematoma foi a complicação mais frequente.
\end{abstract}

Descritores: Neoplasias da Glândula Tireoide. Carcinoma Papilar. Nódulo da Glândula Tireoide. Carcinoma Papilar. Variante Folicular. Tireoidectomia.

\section{INTRODUÇÃO}

$\mathrm{O}$ carcinoma bem diferenciado da tireoide (CBDT) é responsável por $90 \%$ das neoplasias malignas da tireoide. Os subtipos papilífero e folicular são os mais frequentes e, em regiões com a ingesta regular de iodo, representam, respectivamente, $80 \%$ e $10 \%$ de todos os carcinomas da tireoide ${ }^{1-3}$. Estudos mostram que $85 \%$ dos pacientes portadores de CBDT apresenta um bom prognóstico independente da abordagem terapêutica adotada. Por outro lado, cerca de $5 \%$ dos pacientes irá evoluir para óbito, não importando a agressividade do tratamento instituído. Enquanto isso, cerca de 10\% dos casos de CBDT terá sua evolução e seu prognóstico diretamente relacionados às medidas terapêuticas recebidas. Justamente neste último grupo é que os fatores preditivos têm maior importância na definição da conduta terapêutica e no aumento de sobrevida4.

A cirurgia do câncer de tireoide é parte importante de uma abordagem de tratamento multidisciplinar. A operação deve estar embasada em recomendações da literatura e deve ser traçado um plano de acompanhamento do paciente pela equipe que está conduzindo o caso. A iodoterapia é um tratamento complementar utilizado de maneira adjuvante no carcinoma bem diferenciado da tireoide, possibilitando a eliminação de focos neoplásicos microscópicos ${ }^{3}$.

Nosso objetivo foi conhecer o perfil epidemiológico dos pacientes submetidos ao tratamento cirúrgico do CBDT e os resultados oncológicos (recidiva e óbito) no seguimento dos pacientes submetidos a tratamento em nossa Instituição. 


\section{MÉTODOS}

Foram analisados laudos citopatológicos e histopatológicos de pacientes submetidos ao tratamento cirúrgico com o procedimento de tireoidectomia (total ou parcial) associada ou não ao esvaziamento cervical no mesmo tempo cirúrgico, selecionados no arquivo de registro eletrônico do Serviço de Anatomia Patológica do Hospital Universitário Cassiano Antônio Moraes (HUCAM) da Universidade Federal do Espírito Santo (UFES) e análise de prontuários médicos arquivados no Serviço de Arquivo Médico e Estatístico (SAME) do HUCAM/UFES no período de janeiro de 2008 a dezembro de 2015. Foram incluídos no estudo pacientes com mais de 18 anos de idade portadores de neoplasia maligna do tipo CBDT (papilífero e folicular) submetidos a tratamento cirúrgico.

Os prontuários foram analisados quanto à distribuição epidemiológica, resultado do tratamento cirúrgico, complicações e fatores prognósticos: sexo, idade, tipo histológico, tamanho do tumor, estadiamento, tipo de tratamento, tempo médio de internação, complicações pós-operatórias, iodoterapia complementar, recidiva/falha de tratamento e seguimento oncológico, remoção inadvertida da glândula paratireoide e tempo médio de espera entre a primeira consulta com o cirurgião de Cabeça e Pescoço e a realização do tratamento cirúrgico.

O Serviço de Anatomia Patológica o HUCAM começou a utilizar a classificação de Bethesda somente em 2011. Dessa forma os laudos citopatológicos de 2011 a 2015 foram reclassificados utilizando-se o mesmo sistema de laudos até 2010, a saber: insatisfatório, benigno, indeterminado ou maligno.

Os pacientes que apresentavam laudo citopatológico de carcinoma papilífero ou folicular da tireoide ou classificação de Bethesda VI foram reclassificados como resultado citopatológico maligno. Os pacientes classificados como bócio coloide, cisto coloide, classificação de Bethesda II, bócio nodular hiperplásico e proliferação folicular hipercelular foram reclassificados como resultado citopatológico benigno. Os pacientes que apresentavam laudo citopatológico de padrão folicular com ou sem a especificação de presença de atipias foram reclassificados como resultado citopatológico indeterminado.

Uma análise descritiva dos dados foi realizada considerando as seguintes variáveis: idade em anos, sexo, presença de linfonodo comprometido pela neoplasia, presença de paratireoide na peça cirúrgica, estadiamento pT, estadiamento pN, estágio TNM, tipo de cirurgia, resultado da citopatologia na punção aspirativa por agulha fina (PAAF), tipo histológico do tumor bem diferenciado, neoplasia maligna do tipo microcarcinoma, tempo de internação, presença de complicação pósoperatória e tipo de complicação, quando presente, tempo desde o diagnóstico até o tratamento cirúrgico em meses, realização de iodoterapia pós-operatória, presença de recidiva da doença e óbito pela doença.

A análise trouxe um panorama da tendência central e dispersão dos dados através dos gráficos box-plot e da estimação da média, variância, desvio padrão e mediana, e de sua distribuição através da elaboração de tabelas e outros gráficos. Para análise de regressão logística univariada, algumas variáveis foram categorizadas: o valor de idade em anos foi dividido em duas categorias (idade menor do que 45 anos ou igual ou maior que 45 anos); o tipo de cirurgia foi categorizado em três categorias (tireoidectomia parcial, tireoidectomia total e tireoidectomia total com esvaziamento cervical); o tipo histológico de tumor bem diferenciado foi dividido em duas categorias (carcinoma papilífero e carcinoma folicular); a iodoterapia pósoperatória foi avaliada segundo três categorias (realizada, não indicada, e indicada, mas não realizada).

Todos os cálculos e testes estatísticos foram realizados nos software Statsoft STATISTICA 10 e IBM SPSS 22 e foram considerados significativos estatisticamente os valores de $p<0,05$.

O trabalho foi aprovado previamente pelo Comitê de Ética da Instituição, tendo o parecer no 38642214.5.0000.5071. 


\section{RESULTADOS}

No período estudado, 353 pacientes foram submetidos ao tratamento cirúrgico da glândula tireoide. Após a aplicação dos critérios de inclusão, foram selecionados 99 casos de carcinoma bem diferenciado da tireoide. Destes, quatro foram excluídos por ausência de registro de seguimento pós-operatório. Dos 95 pacientes estudados, oito $(8,42 \%)$ eram do sexo masculino e $87(91,58 \%)$ do sexo feminino. A média de idade foi de 50,2 anos, com mediana de 49 anos, variando de 23 a 81 anos. A faixa etária abaixo de 45 anos ocorreu em 37,9\% dos pacientes e a igual ou superior a 45 anos em $62,1 \%$ dos pacientes.

Com relação ao tipo histológico, 87 pacientes $(91,6 \%)$ eram portadores de carcinoma papilífero de tireoide, sendo 23 considerados microcarcinomas, ou seja, tumores menores do que $1 \mathrm{~cm}$, e oito pacientes $(8,4 \%)$ portadores de carcinoma folicular, sendo um paciente portador de carcinoma de células de Hürthle, uma variante mais agressiva do carcinoma folicular.

O laudo citopatológico da PAAF realizada no pré-operatório pôde ser obtida no registro eletrônico do Serviço de Anatomia Patológica em 92 pacientes. Com exceção dos pacientes com diagnóstico de neoplasia maligna na PAAF, que totalizaram 50 casos (54,36\%), todos os demais resultados citopatológicos (45,64\%) foram reclassificados. Em 20 (21,7\%) pacientes o resultado foi de neoplasia benigna, em 22 (23,9\%), o resultado foi indeterminado e em 50 (54,4\%), foi positivo para carcinoma bem diferenciado, como pode ser observado na figura 1. Dos 20 pacientes que tiveram PAAF de neoplasia benigna, nove (45\%) apresentavam microcarcinoma papilífero. Dos 22 pacientes que tinham PAAF com resultado citopatológico indeterminado, sete $(31,8 \%)$ apresentavam microcarcinoma papilífero.

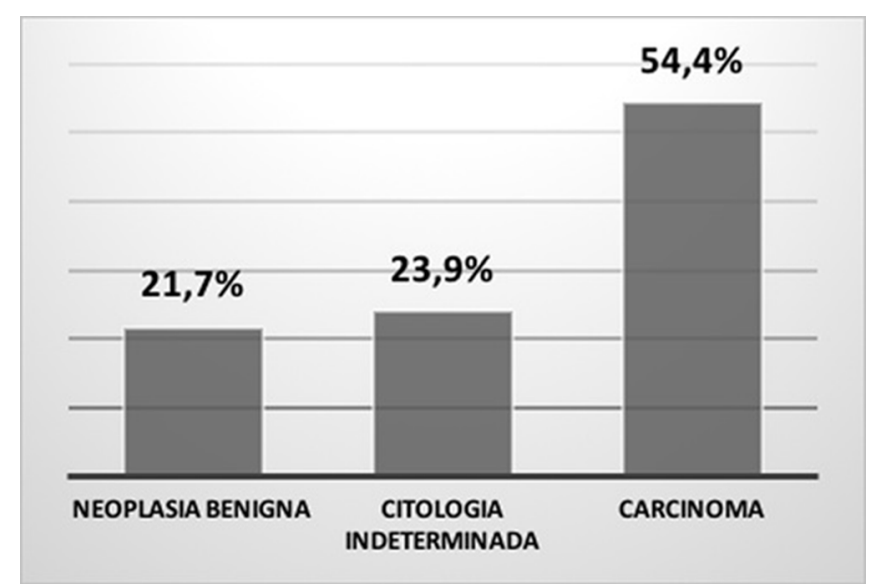

Figura 1. Distribuição por resultado citopatológico da PAAF dos pacientes submetidos ao tratamento cirúrgico por CBDT no HUCAM entre 2008 e 2015 em valores relativos.

Doze pacientes $(12,6 \%)$ foram submetidos à tireoidectomia parcial como tratamento inicial, com diagnóstico de microcarcinoma em cinco, de carcinoma papilífero variante folicular encapsulada em dois e de carcinoma papilífero variante clássica em cinco. Apenas os pacientes portadores de microcarcinoma não foram submetidos a novo procedimento cirúrgico com totalização de tireoidectomia. Sessenta e dois pacientes $(65,3 \%)$ foram submetidos à tireoidectomia total e 21 pacientes $(22,1 \%)$ foram submetidos à tireoidectomia total associada a esvaziamento cervical (oito pacientes foram submetidos a apenas esvaziamento do compartimento central, dez pacientes submetidos a esvaziamento de compartimento central associado a níveis II a V unilateral e três pacientes a esvaziamento de compartimento central associado a níveis II a V bilateralmente).

O tempo médio de internação foi de 2,24 dias, variando de um a 16 dias. As complicações pósoperatórias ocorreram em seis pacientes, sendo uma complicação cirúrgica (hematoma, que foi submetido à reoperação com drenagem e revisão de hemostasia)e cinco complicações clínicas (uma hipertermia maligna e quatro arritmias cardíacas do tipo fibrilação atrial). Nenhum destes pacientes morreu ou teve sequelas das complicações. A média do tempo de internação dos pacientes sem complicações foi de 1,93 dias e dos pacientes com complicações de 6,83 dias. 
A remoção inadvertida de uma glândula paratireoide ocorreu em cinco pacientes, um submetido à tireoidectomia parcial, dois à tireoidectomia total e dois à tireoidectomia total associada a algum esvaziamento cervical. Nenhum paciente teve mais de uma glândula paratireoide removida inadvertidamente. Nenhum destes pacientes evoluiu com hipoparatireoidismo pósoperatório por mais de 30 dias.

O estadiamento $\mathrm{T}$ dos tumores foi pT1a em 24 pacientes ( 23 carcinomas papilíferos e um carcinoma folicular), pT1b em 16, pT2 em 11, pT3 em 42 e pT4 em dois. O estadiamento $\mathrm{N}$ foi pN0 em 75 casos, pN1a em oito e pN1b em 12. Ao aplicarmos os critérios de estadiamento do sistema TNM que utiliza os grupos etários na classificação, encontramos 67 pacientes estádio I, quatro pacientes estádio II, 20 pacientes estádio III e quatro pacientes estádio IVA (Figura 2).

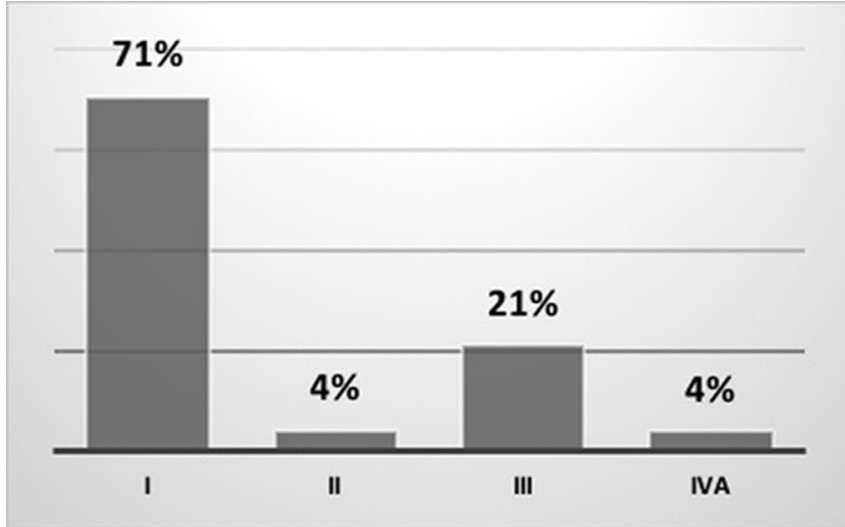

Figura 2. Distribuição por estadiamento pelo sistema TNM dos pacientes submetidos ao tratamento cirúrgico por CBDT no HUCAM entre 2008 e 2015 em valores relativos.

O tempo médio de seguimento pósoperatório foi de 36,9 meses, variando de dois a 91. Recidiva foi observada em quatro pacientes. Um paciente apresentou recidiva linfonodal 14 meses após a cirurgia inicial e foi submetido a esvaziamento cervical, um paciente apresentou recidiva no leito tireoideano um ano após a cirurgia inicial e foi submetido à cervicotomia exploradora e esvaziamento recorrencial, um paciente apresentou recidiva linfonodal em mediastino quatro meses depois e foi submetido a esvaziamento de mediastino anterossuperior e um paciente apresentou recidiva em nível $\vee$ quatro meses depois e foi submetido a esvaziamento cervical seletivo em nível $V$. Todos os pacientes encontram-se em acompanhamento ambulatorial regular sem evidência de doença (Figura 3).

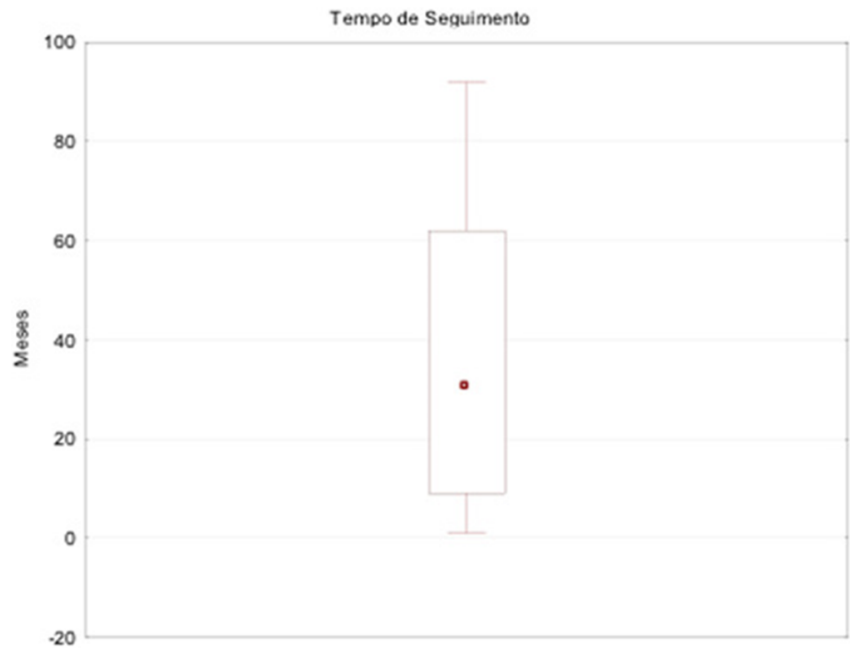

Figura 3. Distribuição por tempo de seguimento em meses completos dos pacientes submetidos ao tratamento cirúrgico por CBDT no HUCAM entre 2008 e 2015.

Com relação aos fatores prognósticos estudados, como sexo, idade acima de 45 anos, tamanho do tumor (estágio pT), acometimento linfonodal (estágio pN), estadiamento da doença, tipo de tratamento cirúrgico, tumor do tipo microcarcinoma, tipo histológico e realização de iodoterapia pós-operatória, obtivemos os resultados expressos na figura 4.

A análise univariada dos fatores prognósticos não mostrou significância estatística, e pode ser avaliada na tabela 1.

\section{DISCUSSÃO}

O carcinoma bem diferenciado da tireoide é a neoplasia endócrina mais frequente e sua incidência e prevalência estão em ascensão. Dados do SEER ${ }^{5}$ mostram um crescimento dos casos novos de câncer de tireoide ao longo das ultimas décadas, com uma estimativa de 13,9 novos casos por 


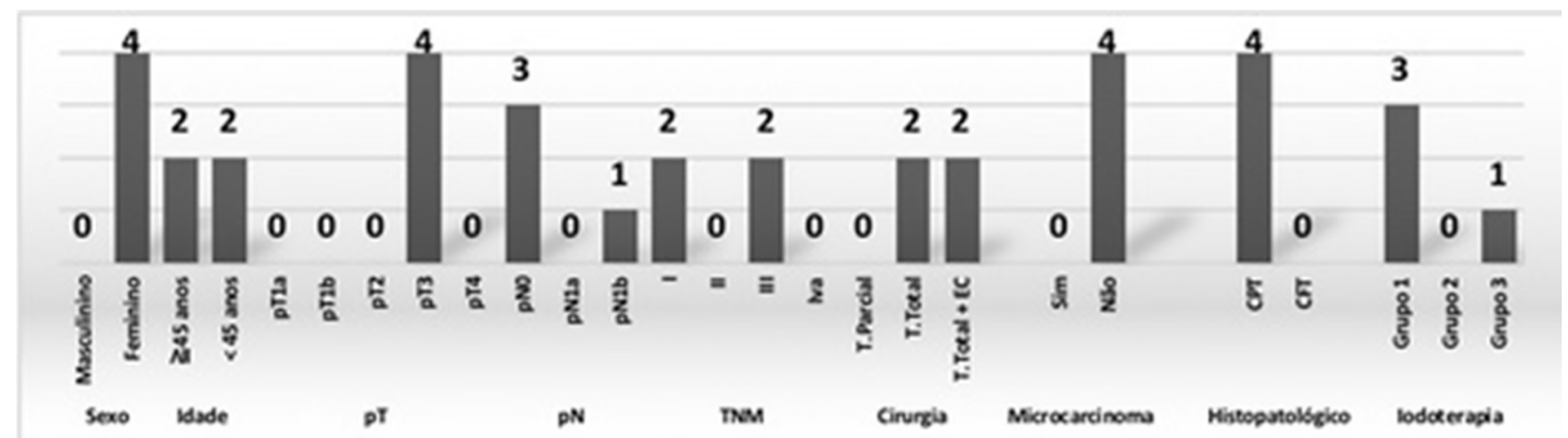

Figura 4. Distribuição de recidiva dos pacientes submetidos ao tratamento cirúrgico por CBDT no HUCAM entre 2008 e 2015 em valores absolutos.

Tabela 1. Análise univariada dos fatores prognósticos para recidiva do carcinoma bem diferenciada da tireoide.

\begin{tabular}{cc}
\hline Fator prognóstico & $\mathrm{p} \mathrm{valor}{ }^{\star}$ \\
\hline Idade acima de 45 anos & 0,962 \\
Estadiamento pT & 0,151 \\
Estadiamento pN & 0,684 \\
Estadio (TNM) & 0,415 \\
Tipo de cirurgia & 0,433 \\
Tumor <1cm & 0,424 \\
Tipo histológico & 0,575 \\
lodoterapia & 0,619 \\
\hline
\end{tabular}

* Teste Qui-quadrado.

100.000 habitantes, em 2016, o que corresponde a $3,8 \%$ de todos os novos casos de câncer nos EUA ${ }^{5,6}$. No Brasil, dados do INCA, estimam uma incidência de 5,78 novos casos a cada 100.000 habitantes $^{7}$.

Dados epidemiológicos americanos revelam que a incidência do carcinoma bem diferenciado da tireoide é cerca de quatro vezes maior em mulheres do que em homens 5 . Em nosso estudo, a relação foi de 10:1. As mulheres tendem a realizar consultas médicas com maior frequência ao longo de sua vida quando comparadas aos homens. Ultrassonografias, muitas vezes solicitadas de maneira rotineira por clínicos ou ginecologistas, têm aumentado o diagnóstico de nódulos tireoideanos em mulheres, o que pode explicar o aumento também de detecção de câncer de tireoide nestes nódulos, especialmente os tumores iniciais. A mediana de idade de 49 anos se aproxima dos dados do SEER ${ }^{5}$, que foi de 51 anos.
Agrupando os pacientes por faixa etária em idade houve uma distribuição bastante semelhante aos dados epidemiológicos estabelecidos ${ }^{5-7}$.

O teste diagnóstico mais comumente utilizado para o diagnóstico dos nódulos tireoidianos é a punção aspirativa por agulha fina (PAAF). A citologia da PAAF tem elevada acurácia em diagnóstico de nódulos benignos ou malignos na maioria dos casos, entretanto, até $10 \%$ a $40 \%$ das amostras de PAAF são diagnosticadas como indeterminados para malignidade, frequentemente tendo o diagnóstico de malignidade apenas no histopatológico ${ }^{8}$. A acurácia diagnóstica da PAAF para nódulos tireoidianos pode ser afetada pelo tamanho do tumor, com taxas de falso negativos para nódulos maiores do que $3 \mathrm{~cm}$ a $4 \mathrm{~cm}$, chegando até $30 \% 9,10$. A positividade para carcinoma na PAAF ocorreu em $54,4 \%$ dos pacientes em nosso estudo. A incidência de tumores menores do que $1 \mathrm{~cm}$ foi elevada (25,3\%), assim como, o número de punções com resultado indeterminado (23,9\%).

O tipo histopatológico mais comum foi o carcinoma papilífero $(91,6 \%)$. Dados mostram que cerca de $88 \%$ dos tumores bem diferenciados da tireoide são carcinoma papilífero e 12\% de carcinoma folicular ${ }^{8,11,12}$. A incidência de microcarcinoma foi de 25,3\%, e estudos têm mostrado uma distribuição de $6 \%$ a $28 \%$ de microcarcinomas nos tumores bem diferenciados. A incidência de microcarcinoma, no Japão, chega a $28,4 \%$ dos casos, e em países, como a Colômbia, é de cerca de 5,6\%. 
A disponibilidade maior de exames diagnósticos com resolução de imagem maior e a possibilidade de punções aspirativas guiadas por USG podem justificar esta maior taxa de detecção de tumores menores de $1 \mathrm{~cm}^{6,13,14}$.

O tratamento cirúrgico do CBDT permanece controverso. Alguns estudos endossavam a tireoidectomia total como abordagem cirúrgica inicial para o CBDT maior do que $1 \mathrm{~cm}$ de diâmetro ${ }^{15}$, baseados em dados retrospectivos que sugeriam que o procedimento cirúrgico bilateral permitia uma melhor sobrevida do paciente ${ }^{16}$, diminuía as taxas de recidiva ${ }^{17,18}$, permitia, como rotina, o uso da iodoterapia complementar e facilitava a detecção de doença recorrente/residual durante o seguimento. Entretanto, dados recentes têm demonstrado que, em pacientes selecionados, a resposta clínica é muito similar em pacientes submetidos à tireoidectomia parcial ou total ${ }^{19-23}$. Estudos japoneses mostram que, em pacientes de baixo risco e de muito baixo risco, a vigilância ativa sem tratamento cirúrgico como opção terapêutica inicial pode ser uma opção segura, sendo a tireoidectomia reservada para pacientes que apresentam sinais de progressão de doença ${ }^{24}$.

No Brasil, a recomendação padrão para tratamento dos tumores bem diferenciados de tireoide é a tireoidectomia total ${ }^{25}$. A tireoidectomia parcial pode ser indicada para doença unifocal menor de $1 \mathrm{~cm}$ (microcarcinoma), sem acometimento linfonodal ou extravasamento capsular. Em nosso estudo, 12 pacientes foram submetidos à tireoidectomia parcial como tratamento inicial. Destes, cinco apresentavam microcarcinoma e não foram submetidos à totalização de tireoidectomia. Todos os demais pacientes foram tratados com tireoidectomia total e, em 21 pacientes, o esvaziamento cervical foi associado.

Optamos por adotar apenas o sistema de estadiamento patológico de acordo com a AJCC, 7ạ edição ${ }^{24}$, e não o estadiamento clínico porque $21,7 \%$ dos pacientes não apresentava exame citopatológico de neoplasia maligna, mas teve o diagnóstico de neoplasia maligna após o exame histopatológico. Além disso, muitas vezes, a tireoide pode ter um nódulo volumoso e apenas uma pequena parte deste nódulo corresponde a volume tumoral $^{9}$. Recente publicação da AJCC acerca do estadiamento TNM, 8a edição, reclassifica os fatores prognósticos do carcinoma bem diferenciado com relação à idade dos pacientes, aumentando o ponto de corte de 45 para 55 anos.

O acometimento linfonodal ocorreu em $21 \%$ dos pacientes, o que é corroborado por estudos que mostram uma incidência de metástase linfonodal em 15\% a 30\% dos pacientes ${ }^{26,27}$, embora em alguns estudos possa chegar a 47,5\%28. Vários estudos mostram que a presença de metástase linfática de carcinoma bem diferenciado de tireoide não altera a sobrevida global dos pacientes, no entanto, os índices de recidiva e de persistência de doença podem ser maiores em pacientes com acometimento linfático 4,29,30. Na presença de metástases no compartimento central deve ser realizado o esvaziamento cervical seletivo recorrencial bilateral e de mediastino ânterosuperior ${ }^{24,31}$. Nos pacientes em que é detectada, clinicamente ou através do exame de imagem, a presença de linfonodo cervical em nível fora do compartimento central, é indicada a realização do esvaziamento cervical dos níveis II a V24,31.

A iodoterapia complementar não é rotineiramente recomendada após tireoidectomia para pacientes de baixo risco ${ }^{24}$, ou para aqueles portadores de microcarcinoma submetidos à tireoidectomia parcial ou total. A iodoterapia complementar é indicada para pacientes de risco intermediário e alto risco ${ }^{24}$. Em nosso estudo, a iodoterapia não foi indicada em $26,3 \%$ dos pacientes, foi realizada em $44,2 \%$ e foi indicada, mas ainda não havia sido realizada, em 21,1\% dos casos. A indicação de iodoterapia complementar não foi realizada nos pacientes portadores 
de microcarcinomas que não apresentavam invasão capsular ou acometimento linfonodal, considerados, portanto, pacientes de muito baixo risco para recidiva da doença. Em um paciente não indicamos a iodoterapia complementar pelo fato de o paciente ter alergia a iodo.

Os principais fatores prognósticos relacionados à recidiva e óbito pela doença são: idade maior do que 45 anos, presença de metástase à distância, extensão extratireoideana, tamanho do tumor maior do que $4 \mathrm{~cm}$, sexo masculino e tipo histológico folicular ${ }^{4,15,24,32}$. A recidiva do carcinoma da tireoide ocorreu em 4,65\% dos pacientes. No entanto, não houve relação com nenhum dos fatores prognósticos analisados. Estes resultados certamente refletem o número pequeno de recidivas.

A complicação cirúrgica pós-operatória mais frequente foi o hematoma, ocorrendo em 1,05\% dos pacientes. Os estudos mostram que a complicação hemorrágica é a mais frequente. ocorrendo em $0,4 \%$ a $4 \%$ dos casos. A glândula tireoide é muito vascularizada e isto aumenta a ocorrência de complicações hemorrágicas no pós-operatório ${ }^{10,33,34}$.

A remoção inadvertida da glândula paratireoide é uma das possíveis complicações pósoperatórias e isto pode causar hipoparatireoidismo, que está presente em $1 \%$ a $5 \%$ dos pacientes submetidos à tireoidectomia ${ }^{35}$. Dados na literatura revelam uma incidência de remoção inadvertida da glândula paratireoide em 6,4\% a 16,4\% ${ }^{35}$. Aincidência da remoção inadvertida da glândula paratireoide, em nosso estudo, ocorreu em cinco (5,26\%) pacientes. A extensão do tratamento cirúrgico não influenciou na retirada da glândula paratireoide.

Observamos, com nosso estudo, que o carcinoma papilífero da tireoide foi a neoplasia maligna mais frequente, acometendo o sexo feminino na faixa etária dos 49 anos mais frequentemente. A recidiva locorregional ocorreu em quatro pacientes e o hematoma foi a complicação mais frequente. Nosso tempo de seguimento foi curto, o que limita nossas conclusões acerca dos resultados oncológicos.

\title{
A B S T R A C T
}

\begin{abstract}
Objective: to know the epidemiological profile of patients undergoing surgery for well-differentiated thyroid carcinoma at the Cassiano Antônio Moraes University Hospital of the Federal University of Espírito Santo, as well as the oncological results and the main postoperative complications. Methods: we conducted a cross-sectional, retrospective study of patients with well-differentiated thyroid carcinoma (WDTC) operated from January 2008 to December 2015. Results: During the study period, 95 of the 353 patients undergoing surgical treatment of the thyroid gland had WDTC. Papillary carcinoma was the most frequent (91.57\%). Total thyroidectomy not associated with cervical emptying was the most frequent surgical procedure (65.26\%). Postoperative complications occurred in $6.31 \%$ of patients, hematoma being the most frequent. The mean follow-up time was 36.9 months. Relapse occurred in four patients (4.21\%), being locoregional in all cases. The prognostic factors analyzed, such as gender, age, tumor size, lymph node involvement, staging, type of surgery, histology and complementary iodine therapy did not show statistical significance. Conclusion: papillary carcinoma was the most common thyroid malignant neoplasm, affecting women in the 49-year-old age group more frequently. Loco-regional recurrence occurred in four patients. Hematoma was the most frequent complication.
\end{abstract}

Keywords: Thyroid Neoplasms. Carcinoma. Papillary. Thyroid Nodule. Carcinoma. Papillary. Follicular. Thyroidectomy.

\section{REFERÊNCIAS}

1. Kumar V, Abbas AK, Fausto N, Aster JC, editores. Robbins \& Cotran Patologia - bases patológicas das doenças. 8a ed. Nova lorque: Saunders Elsevie; 2010.

2. Vanderpump MP. The epidemiology of thyroid disease. Br Med Bull [Internet]. 2011 Jan 1 [cited 2015 Dec 6];99(1):39-51. Available from: http:// bmb.oxfordjournals.org/content/99/1/39.abstract
3. Figueiredo EM, Monteiro M, Ferreira A. Tratado de Oncologia. 1a ed. Rio de Janeiro: Revinter; 2013.

4. Shaha AR. Implications of prognostic factors and risk groups in the management of differentiated thyroid cancer. Laryngoscope. 2004; 114(3):393402.

5. Howlader N, Noone AM, Krapcho M, Miller D, Bishop K, Altekruse SF, Kosary CL, Yu M, Ruhl J, Tatalovich Z, Mariotto A, Lewis DR, Chen HS, Feuer EJ, Cronin 
KA, editors. SEER Cancer Statistics Review, 1975-2013 [Internet]. Bethesda, MD: National Cancer Institute; 2016 [cited 2015 Dec 6]. Available from: http://seer. cancer.gov/csr/1975_2013/

6. Davies $L$, Welch $H G$. Increasing incidence of thyroid cancer in the United States, 1973-2002. JAMA. 2006;295(18):2164-7.

7. Brasil. Ministério da Saúde. Instituto Nacional de Câncer José Alencar Gomes da Silva. Estimativa 2016. Incidência de câncer no Brasil. Rio de Janeiro: INCA, 2016.

8. Pereira JA, Jimeno J, Miquel J, Iglesias M, Munné A, Sancho JJ, et al. Nodal yield, morbidity, and recurrence after central neck dissection for papillary thyroid carcinoma. Surgery. 2005;138(6):1095101.

9. Samona S, Hagglund K, Edhayan E. Case cohort study of risk factors for post-thyroidectomy hemorrhage. Am J Surg. 2016;211(3):537-40.

10. Lee HS, Lee BJ, Kim SW, Cha YW, Choi YS, Park YH, et al. Patterns of post-thyroidectomy hemorrhage. Clin Exp Otorhinolaryngol. 2009;2(2):72-7.

11. Wartofsky L, Van Nostrand D, editors. Thyroid cancer: a comprehensive guide to clinical management. Springer Science \& Business Media; 2007.

12. Tuttle RM, Ross DS, Mulder JE. Differentiated thyroid cancer: Overview of management. UpToDate ${ }^{\circledR}$ [Internet]. 2015; (table 1):1-29. Available from: http://www.uptodate.com

13. Fukunaga FH, Yatani R. Geographic pathology of occult thyroid carcinomas. Cancer. 1975;36(3):1095-9.

14. Ito $Y$, Miyauchi A. A therapeutic strategy for incidentally detected papillary microcarcinoma of the thyroid. Nat Clin Pract Endocrinol Metab. 2007;3(3):240-8.

15. American Thyroid Association (ATA) Guidelines Taskforce on Thyroid Nodules and Differentiated Thyroid Cancer, Cooper DS, Doherty GM, Haugen $\mathrm{BR}, \mathrm{Kloos} \mathrm{RT}$, Lee SL, Mandel SJ, Mazzaferri EL, Mclver B, Pacini F, Schlumberger M, Sherman SI, Steward DL, Tuttle RM. Revised American Thyroid Association management guidelines for patients with thyroid nodules and differentiated thyroid cancer. Thyroid. 2009;19(11):1167-214.
16. Bilimoria $K Y$, Bentrem DJ, Ko $C Y$, Stewart $A K$, Winchester DP, Talamonti MS, et al. Extent of surgery affects survival for papillary thyroid cancer. Ann Surg [Internet]. 2007 Sep [cited 2015 Dec 5];246(3):375-84. Available from: https://www.ncbi.nlm.nih.gov/pmc/ articles/PMC 1959355/pdf/20070900s00004p375.pdf

17. Grant CS, Hay ID, Gough IR, Bergstralh EJ, Goellner $J R$, McConahey MW. Local recurrence in papillary thyroid carcinoma: is extent of surgical resection important? Surgery. 1988;104(6):954-62.

18. Hay ID, Grant CS, Bergstralh EJ, Thompson GB, Van Heerden JA, Goellner JR, et al. Unilateral total lobectomy: Is it sufficient surgical treatment for patients with AMES low-risk papillary thyroid carcinoma? Surgery [Internet]. 1998 Dec [cited 2015 Dec 5];124(6):958-66. Available from: http://www.scopus.com/inward/record. url?eid=2-s2.0-0031762998\&partnerlD=tZOtx3y1

19. Matsuzu K, Sugino K, Masudo K, Nagahama M, Kitagawa W, Shibuya $\mathrm{H}$, et al. Thyroid lobectomy for papillary thyroid cancer: long-term follow-up study of 1,088 cases. World J Surg. 2014;38(1):68-79.

20. Barney BM, Hitchcock YJ, Sharma P, Shrieve DC, Tward JD. Overall and cause-specific survival for patients undergoing lobectomy, near-total, or total thyroidectomy for differentiated thyroid cancer. Head Neck. 2011;33(5):645-9.

21. Nixon IJ, Ganly I, Patel SG, Palmer FL, Whitcher MM, Tuttle RM, et al. Thyroid lobectomy for treatment of well differentiated intrathyroid malignancy. Surgery. 2012;151(4):571-9.

22. Mendelsohn AH, Elashoff DA, Abemayor E, St John MA. Surgery for papillary thyroid carcinoma: is lobectomy enough? Arch Otolaryngol Head Neck Surg. 2010;136(11):1055-61.

23. Haigh $\mathrm{Pl}$, Urbach $\mathrm{DR}$, Rotstein LE. Extent of thyroidectomy is not a major determinant of survival in low- or high-risk papillary thyroid cancer. Ann Surg Oncol. 2005;12(1):81-9.

24. Haugen BR, Alexander EK, Bible KC, Doherty GM, Mandel SJ, Nikiforov YE, et al. 2015 American Thyroid Association Management Guidelines for Adult Patients with Thyroid Nodules and Differentiated Thyroid Cancer: The American Thyroid Association Guidelines Task Force on Thyroid Nodules and Differentiated Thyroid Cancer. Thyroid. 2016;26(1):1-133. 
25. Rosário PW, Ward LS, Carvalho GA, Graf H, Maciel RM, Maciel LM, Maia AL, Vaisman M; Sociedade Brasileira de Endocrinologia e Metabologia. Thyroid nodules and differentiated thyroid cancer: update on the Brazilian consensus. Arq Bras Endocrinol Metab. 2013;57(4):240-64. English, Portuguese.

26. Gimm O, Rath FW, Dralle H. Pattern of lymph node metastases in papillary thyroid carcinoma. $\mathrm{Br} \mathrm{J}$ Surg. 1998;85(2):252-4.

27. El Foll HA, El-Sebaey HI, El-Kased AF, Hendawy A, Kamel MM. Pattern and distribution of lymph node metastases in papillary thyroid cancer. J Clin Exp Pathol [Internet]. 2015 Jan [cited 2017 Dec 5];5(1):1-6. Available from: http://www.omicsonline. org/open-access/pattern-and-distribution-oflymph-node-metastases-in-papillary-thyroidcancer-2161-0681.1000204.php?aid=37339

28. Shaha AR, Shah JP, Loree TR. Patterns of nodal and distant metastasis based on histologic varieties in differentiated carcinoma of the thyroid. Am J Surg. 1996;172(6):692-4.

29. Mazzaferri EL, Doherty GM, Steward DL. The pros and cons of prophylactic central compartment lymph node dissection for papillary thyroid carcinoma. Thyroid. 2009;19(7):683-9.

30. Pereira JA, Jimeno J, Miquel J, Iglesias M, Munné A, Sancho JJ, et al. Nodal yield, morbidity, and recurrence after central neck dissection for papillary thyroid carcinoma. Surgery. 2005;138(6):1095-100; discussion 1100-1.

31. Callender GG, Carling T, Christison-Lagay E, Udelsman R. Surgery for thyroid cancer. Endocrinol Metab Clin North Am. 2014;43(2):443-58.

32. Nikiforova MN, Nikiforov YE. Molecular diagnostics and predictors in thyroid cancer. Thyroid. 2009; 19(12):1351-61.

33. Frick $T$, Largiadèr $F$. [Perioperative complications in thyroid gland surgery]. Langenbecks Arch Chir.1991;376(5):291-4. German.

34. Lacoste L, Gineste D, Karayan J, Montaz N, Lehuede MS, Girault M, et al. Airway complications in thyroid surgery. Ann Otol Rhinol Laryngol. 1993;102(6):441-6.

35. Khairy GA, Al-Saif A. Incidental parathyroidectomy during thyroid resection: Incidence, risk factors, and outcome. Ann Saudi Med. 2011;31(3):274-8.

Recebido em: 07/06/2018

Aceito para publicação em: 22/08/2018

Conflito de interesse: nenhum.

Fonte de financiamento: nenhuma.

\section{Endereço para correspondência:}

Ricardo Mai Rocha

E-mail: ricardomai@gmail.com ricardomai@yahoo.com.br 